\title{
Fabrication of Thick Periodically-poled Lithium Niobate Crystals by Standard Electric Field Poling and Direct Bonding
}

\author{
Byoung Joo Kim, Chung-Sik Kim, Dong Jin Kim, Hwan Hong Lim, Sung Kyun Park, \\ and Myoungsik Cha* \\ Department of Physics, Pusan National University, Busan 609-735, Korea \\ Kyung Jo Kim \\ School of Electrical Engineering, Pusan National University, Busan 609-735, Korea
}

(Received October 20, 2010 : revised November 4, 2010 : accepted November 24, 2010)

\begin{abstract}
We fabricated $1 \mathrm{~mm}$-thick periodically-poled lithium niobate (PPLN) crystals by using a high-voltage amplifier for standard electric field poling combined with a voltage multiplier. Furthermore, two $1 \mathrm{~mm}$-thick PPLNs were directly bonded to make a 2 mm-thick PPLN. The large aperture allowed broad angular tuning, and a broad spectral range of quasi-phase matched second-harmonic generation can be achieved in a single channel. High-power applications are also expected.
\end{abstract}

Keywords: Periodically-poled lithium niobate, Quasi-phase-matching, Poling

OCIS codes : (190.2620) Harmonic generation and mixing; (160.3730) Lithium niobate

\section{INTRODUCTION}

The quasi-phase-matching (QPM) technique provides not only high efficiency, but also many degrees of freedom in designing nonlinear optical frequency conversion devices [1]. It has been successfully implemented in ferroelectric crystals such as periodically-poled lithium niobate (PPLN) crystals, providing efficient optical second-harmonic generation (SHG) and other quadratic optical parametric processes, due to the large nonlinear optical tensor element $d_{33}$ [2-3]. Although congruent lithium niobate (CLN) crystals are produced with reliable quality [4], the high coercive field allows poling of thin crystals only $(<1 \mathrm{~mm})$. As a result, tight focusing is always needed, limiting their high power or intra-cavity applications. Ferroelectric crystals with low coercive fields have been developed as a solution to this problem, by reducing the microscopic defects which are the origins of the majority of the coercive fields. Thick crystals $(1 \sim 5 \mathrm{~mm})$ of MgO-doped and/or stoichiometric lithium niobate and lithium tantalate have been utilized in high-power QPM applications [5-6].

However, some problems still remain with the newlydeveloped ferroelectric crystals: (1) poling is not easy, sometimes producing low-quality QPM devices, (2) the reversed domains can relax due to the low coercive field, and (3) the uniformity of the domain width throughout the thickness is not as good as PPLNs made of CLN. Missey et al. reported diffusion bonding of three $1 \mathrm{~mm}$-thick PPLNs, producing a large aperture PPLN for optical parametric oscillator [7]. In their work, the CLN stacks were pressed and heated at high temperature during bonding. Directbonding of various materials has been investigated for fabricating optical devices such as waveguides [8]. A buried waveguide was made by direct-hetero-bonding of lithium niobate and lithium tantalate crystals [9], and efficient SHG has been obtained with a similarly fabricated buried PPLN waveguide [10]. In this work, we utilized a similar directbonding method to make a large-aperture PPLN device for the demonstration of "slanted QPM" SHG. We poled 1 mm-thick CLN crystals by a modified electric field poling method, and by directly bonding two of them, we fabricated a 2 mm-thick PPLN. The quality of the bonded PPLN was good enough to perform a slanted QPM SHG experiment by tuning the wave propagation direction between the $\mathrm{x}$-axis and the $\mathrm{z}$-axis within the aperture of the thick PPLN. [Usually QPM is designed for non-critical propagation

\footnotetext{
*Corresponding author: mcha@pnu.edu

Color versions of one or more of the figures in this paper are available online.
} 
(wave vector perpendicular to the $\mathrm{z}$ - axis)].

\section{ELECTRIC FIELD POLING OF 1 mm-CLN}

We used the standard electric field poling technique [11] for poling $1 \mathrm{~mm}$-thick CLN. However, the coercive field of CLN is $\sim 22 \mathrm{kV} / \mathrm{mm}$, while the maximum capacity of our high-voltage power amplifier is only \pm 20 $\mathrm{kV}$ (TREK, 20/20B). We added a voltage-multiplier circuit in order to generate successive pulses of $-5 \mathrm{kV}$ and +17 $\mathrm{kV}$ [12], providing $22 \mathrm{kV}$ to a z-cut $1 \mathrm{~mm}-\mathrm{CLN}$ substrate (Crystal Technology), as described in Fig. 1. The QPM period was $18.6 \mu \mathrm{m}$, the channel width was $3.0 \mathrm{~mm}$ (defined by a photomask), and the channel length was 15 $\mathrm{mm}$ along the $\mathrm{x}$-axis.

\section{DIRECT BONDING OF TWO 1 mm-PPLN CRYSTALS}

We directly bonded the two $1 \mathrm{~mm}$-PPLNs, making a 2 mm-thick PPLN. First, the $1 \mathrm{~mm}-\mathrm{PPLNs}$ were cleaned and chemically treated in a $\mathrm{H}_{2} \mathrm{O}_{2}: \mathrm{NH}_{4} \mathrm{OH}: \mathrm{H}_{2} \mathrm{O} \quad(1: 1: 6)$ solution in order to form a hydrophilic OH-layer on the surfaces for bonding (z-faces) [13]. The two PPLNs were aligned (+ domains on top of +domains, etc.), and gently pressed in DI-water, followed by an annealing process at $700{ }^{\circ} \mathrm{C}$ for an hour. After annealing, the bonding was so strong that the original PPLNs could not be separated. Photographs of the bonded sample are shown in Fig. 2.

We verified excellent alignment between the ferroelectric domains in the top $1 \mathrm{~mm}$-PPLN and the ones in the bottom PPLN throughout the bonded region as demonstrated in Fig. 2(a). As a result, we could fabricate a PPLN with thickness $2 \mathrm{~mm}$, channel width $3 \mathrm{~mm}$, and length $15 \mathrm{~mm}$, with a only small unbounded region near the edge (Fig. 2(b)).

In order to check the optical loss of the bonding layer quantitatively, we measured the transmission spectrum, which was compared with those of single $1 \mathrm{~mm}$-thick PPLN, and two unbounded $1 \mathrm{~mm}$-thick PPLNs. As shown in Fig. 3, we could conclude that the transmission of the bonded sample $(2 \mathrm{~mm})$ is nearly the same as that of single PPLN in the non-absorbing spectral range between 450 and 1000 $\mathrm{nm}$, indicating that the losses are mostly due to the Fresnel reflections at the air-crystal interfaces. Because the loss at the bonding interface is negligible, we can confirm that the optical quality of the bonded PPLN is excellent.

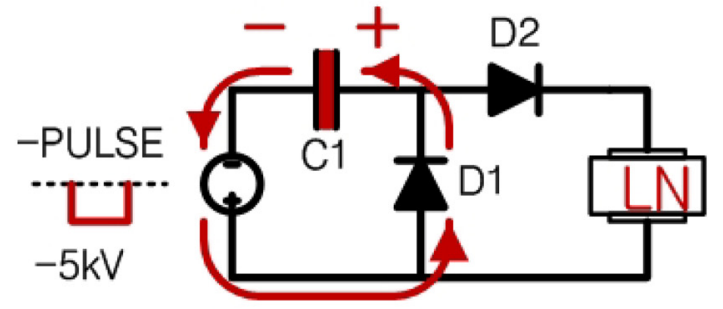

(a)

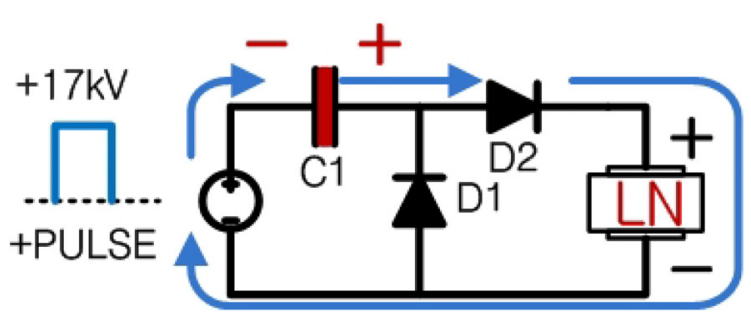

(b)

FIG. 1. Circuit diagram for voltage-multiplier (circle symbol: TREK amplifier, LN: $1 \mathrm{~mm}$-CLN substrate). Diagrams (a) and (b) show the processes of generating a high-voltage pulse $(22 \mathrm{kV})$; Charges of negative pulse $(-5 \mathrm{kV})$ are stored at capacitor $\mathrm{C} 1$ (a), and then positive pulse $(+17 \mathrm{kV})$ of the amplifier is added serially to $\mathrm{C} 1$. A total of $22 \mathrm{kV}$ is applied to LCN. Arrows indicate the directions of electric current.

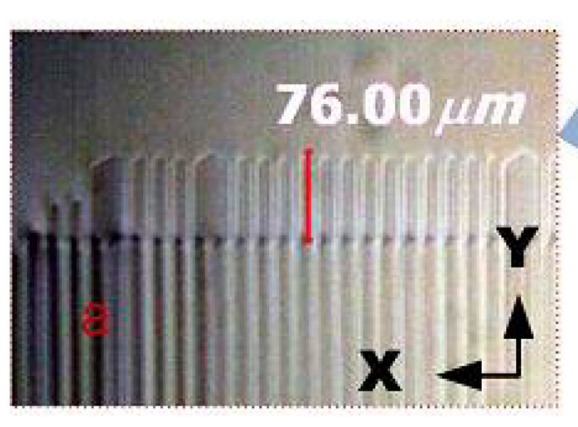

(a)

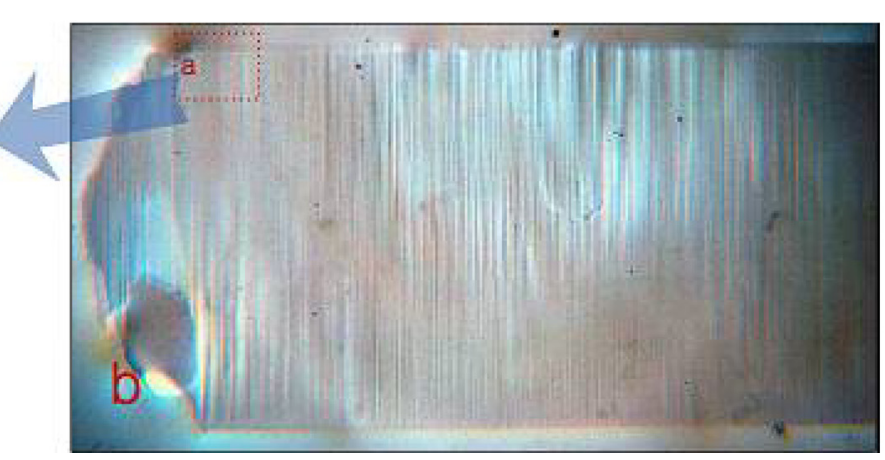

(b)

FIG. 2. Micrographs of the directly bonded PPLN (top view), showing good alignment of ferroelectric domains between top and bottom PPLNs (a), and larger region (lower magnification) of bonded sample (b) (channel width : $3.0 \mathrm{~mm}$ ). Small unbonded area is also observed as indicated with 'b' at a corner. 


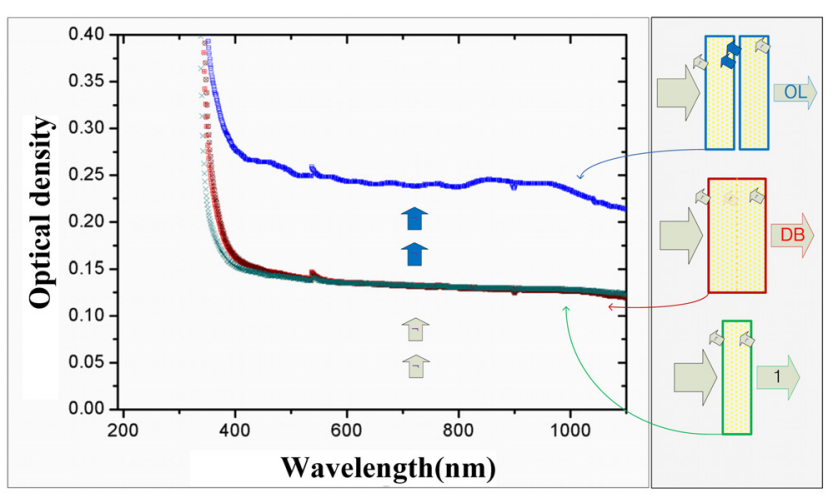

FIG. 3. Transmission spectra (optical density vs. wavelength) for bonded PPLN (red, 2 mm-thick), single 1 mm-thick PPLN (green), and two $1 \mathrm{~mm}$-thick PPLNs (blue, unbounded). Each arrow in graph indicates the loss due to Fresnel reflection at an air-crystal interface.

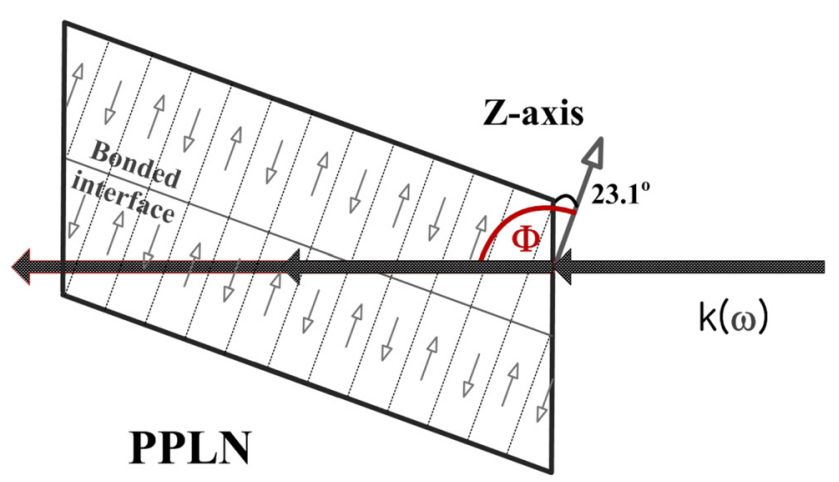

FIG. 4. Configuration of SHG experiment. Both fundamental and harmonic are extraordinary waves (polarized in the plane of paper). $\Phi$ : angle between the optic axis and internal wave vector $\left(=113 \cdot 1^{\circ}\right)$.

\section{SHG EXPERIMENT}

For slanted QPM SHG experiment, we cut and polished the bonded PPLN as shown in Fig. 4. The angle between the optic (z-) axis and the internal wave vector could be changed by rotating the sample in the xz-plane. As a light source, we used a $\beta$-barium borate optical parametric oscillator (OPO) pumped by the third harmonic of a Q-switched Nd:YAG laser. The idler wavelength from the OPO was scanned between $1580 \sim 1640 \mathrm{~nm}$ in order to obtain the SHG-tuning curve at a fixed angle of incidence (normal incidence in this work). The input intensity was attenuated and loosely focused to the sample.

The transmitted second harmonic intensity was measured as a function of fundamental wavelength at a fixed angle of incidence. The result is a typical sinc-square function centered at $806 \mathrm{~nm}$, as shown in Fig. 5. The peak has been confirmed as the first order QPM wavelength by using the Sellmeier's formula of CLN for the extraordinary wave index $n_{e}(\Phi)$ [14]. The first order QPM wavelength is

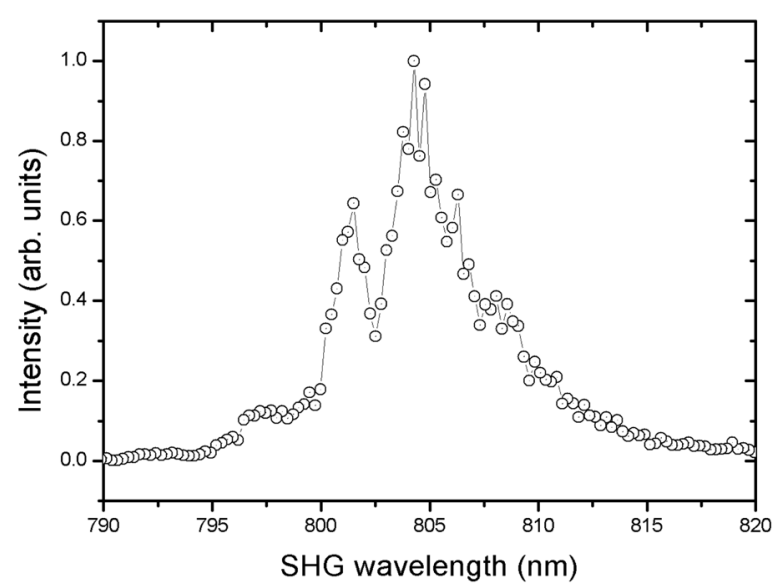

FIG. 5. Slanted QPM SHG intensity versus second-harmonic wavelength; $\Phi=113.1^{\circ}$ (normal incidence).

$767 \mathrm{~nm}$ for conventional PPLN (propagation along x-axis). A large shift of $39 \mathrm{~nm}(767 \mathrm{~nm} \rightarrow 806 \mathrm{~nm})$ has been achieved by slanted QPM. We also note that the results are accurately predictable, due to the well-established Sellmeier formula [14] and reproducible material properties of CLN.

\section{CONCLUSIONS}

We fabricated a $2 \mathrm{~mm}$-thick periodically-poled lithium niobate (PPLN) of high optical quality by direct bonding of two $1 \mathrm{~mm}$-thick PPLNs made of congruent lithium niobate. As an application, we demonstrated "slanted" firstorder QPM SHG by properly choosing the wave propagation direction within the aperture of the thick PPLN. The large aperture allowed broad angular tuning, and a broad spectral range of quasi-phase matched second-harmonic generation can be achieved in a single channel. One can extend the tunable QPM range by stacking more PPLNs, fabricating thicker PPLN crystals. High-power applications such as optical parametric oscillators are also expected.

"This work was supported by the National Research Foundation (NRF) Grant funded by the Ministry of Education, Science and Technology of Korea (2009-0068995)."

\section{REFERENCES}

1. M. M. Fejer, G. A. Magel, D. H. Jundt, and R. L. Byer, "Quasi-phase-matched second harmonic generation: tuning and tolerances," IEEE J. Quantum Electron. 28, 2631-2654 (1992).

2. L. E. Myers, R. C. Eckardt, M. M. Fejer, R. L. Byer, W. R. Bosenberg, and J. W. Pierce, "Quasi-phase matched optical parametric oscillators in bulk periodically poled $\mathrm{LiNbO}_{3}$," J. Opt. Soc. Am. B 12, 2102-2116 (1995).

3. W. R. Bosenberg, A. Drobshoff, J. I. Alexander, L. E. 
Myers, and R. L. Byer, "Continuous-wave singly resonant optical parametric oscillator based on periodically poled $\mathrm{LiNbO}_{3}$," Opt. Lett. 21, 713-715 (1996).

4. K. Pandiyan, Y.-S. Kang, H.-H. Lim, B.-J. Kim, O. Prakash, and M. Cha, "Poling quality evaluation of periodically poled lithium niobate using diffraction method," J. Opt. Soc. Korea 12, 205-209 (2008).

5. H. Ishizuki and T. Taira, "High-energy quasi-phase-matched optical parametric oscillation in a periodically poled $\mathrm{MgO}$ : $\mathrm{LiNbO}_{3}$ device with a $5 \mathrm{~mm}$ X $5 \mathrm{~mm}$ aperture," Opt. Lett. 30, 2918-2920 (2005).

6. T. Hatanaka, K. Nakamura, T. Taniuchi, H. Ito, Y. Furukawa, and K. Kitamura, "Quasi-phase-matched optical parametric oscillation with periodically poled stoichiometric $\mathrm{LiTaO}_{3}$," Opt. Lett. 25, 651-653 (2000).

7. M. J. Missey, V. Dominic, L. E. Myers, and R. C. Eckardt, "Diffusion-bonded stacks of periodically poled lithium niobate," Opt. Lett. 23, 664-666 (1998).

8. J. Haisma, B. A. C. M. Spierings, U. K. P. Biermann, and A. A. van Gorkum, "Diversity and feasibility of direct bonding a survey of a dedicated optical technology," Appl. Opt. 33, 1154-1169 (1994).

9. K. Eda, M. Sugimoto, and Y. Tomita, "Direct heterobonding of lithium niobate onto lithium tantalate," Appl. Phys. Lett. 66, 827-828 (1995).

10. C. B. E. Gawith, D. P. Shepherd, J. A. Abernethy, D. C. Hanna, G. W. Ross, and P. G. R. Smith, "Second-harmonic generation in a direct-bonded periodically poled $\mathrm{LiNbO}_{3}$ buried waveguide," Opt. Lett. 24, 481-483 (1999).

11. G. D. Miller, "Periodically poled lithium niobate: modeling fabrication, and nonlinear-optical performances," Ph.D. Dissertation, Stanford University (1998).

12. A. P. Malvino, Electronic Principles, 5th ed. (McGraw-Hill, New York, USA, 1993).

13. A. Plößl and G. Kräuter, "Wafer direct bonding: tailoring adhesion between brittle materials," Mater. Sci. Eng. R25, 1-88 (1999).

14. D. H. Jundt, "Temperature-dependent Sellmeier equation for the index of refraction, $\mathrm{n}_{\mathrm{e}}$, in congruent lithium niobate," Opt. Lett. 22, 1553-1555 (1997). 